\title{
Estudio clínico y epidemiológico de un brote de beriberi húmedo en Cartagena de Indias, Colombia, 1992-1993
}

\author{
Martínez $M^{1}$, Román $\mathrm{G}^{2}$, de la Hoz $F^{1}$, Podlesky E ${ }^{1}$, Toro $\mathrm{G}^{3}$, Osorio $\mathrm{E}^{1}$, Rodríguez $\mathrm{G}^{1}$, Navarro $\mathrm{L}^{2}$, \\ Tio $\mathrm{FO}^{2}$, Lozano $\mathrm{D}^{4}$, Zúñiga $\mathrm{F}^{4}$, Caballero $\mathrm{B}^{2}$, Spencer $\mathrm{PS}{ }^{2}$, Iglesias $\mathrm{A}{ }^{1}$
}

\begin{abstract}
Resumen
La presente investigación ha tenido como objetivo primordial aclarar la etiología de un cuadro clínico caracterizado por edema de las piernas, disestesias con déficit motor en miembros inferiores que, en dos casos, incluyó una cardiopatía que llevó a insuficiencia cardiaca irreversible. Este brote ocurrió entre julio de 1991 y junio de 1993; afectó selectivamente infantes de marina de la Escuela Naval Almirante Padilla en Cartagena de Indias.
\end{abstract}

La metodología se centró en un estudio de casos y controles en el cual se sometió a cada grupo a un cuidadoso interrogatorio, examen clínico, medición por el laboratorio de metales pesados en sangre y orina de 24 horas y estudio de niveles de tiamina en muestras de suero; se analizaron cuatro biopsias de nervio sural y en el estudio postmortem de los fallecidos se puso especial atención a las alteraciones de miocardio. En muestras ambientales y biológicas, se analizó mercurio, plomo, arsénico y talio.

Los resultados totalizan un grupo de 22 infantes de marina con un cuadro muy uniforme sobre todo por las manifestaciones de una neuropatía periférica nutricional, lo cual sumado a la cardiopatía fatal en dos pacientes, cuyo estudio tanto en microscopia de luz como electrónica, demostró cambios en todo compatibles con lo observado en beriberi, permiten que se considere que el problema de fondo reune las características del beriberi húmedo.

Después de concluir que el problema tenía como fondo un origen nutricional basado, en primer lugar, en una falla de tiamina, se hicieron las recomendaciones y ajustes dietéticos indicados para estos casos con una respuesta inmediata y enteramente satisfactoria.

\section{Summary}

The main goal of this paper has been to understand the etiology of a clinical picture characterized by an edema of the legs and dysesthesia with motor dysfunction of the legs. In two cases the clinical picture ended up in a cardiopathy that lead to an irreversible cardiac failure. This outbreak occurred between July 1991 and June 1993 and affected selectively marines from the Escuela Naval Almirante Padilla in Cartagena the Indias.

\footnotetext{
1 Instituto Nacional de Salud, Santa Fé de Bogotá, Colombia

2 National Institute of Health, Bethesda, Maryland, U.S.A.

3 Universidad Nacional de Colombia, Santa Fé de Bogotá.

4 Hospital Naval de Cartagena, Cartagena.
} 
Our methodology was to carry out a case and control study as follows: ask the patients and their controls to fully answer a questionnaire; to perform clinical exams; to test for heavy metals in blood and 24 hour urine sample and to analyze the levels of thiamine in serum samples. We analyzed four sural nerve biopsies. In the two postmortem examinations we paid special attention to the myocardial changes. In enviromental and biological samples we searched for mercury, lead, arsenic and thalium.

The final outcome of the study was a very uniform picture of the manifestations of a nutritional peripheral neuropathy in a group of 22 marines. This fact plus the two fatal cardiopathies that were studied both by light and electron microscopy showed changes that are compatible with those observed in beriberi. Thus, we concluded that the problem had all the characteristics of wet beriberi.

After we concluded that the source of the problem was a nutritional dificiency, mainly of thiamine, we made the appropiate recommendations and adjustments of the diet indicated in such cases. The response was immediate and entirely satisfactory.

La Escuela Naval Almirante Padilla está ubicada en la isla de Manzanillo, en la ciudad de Cartagena; residen en ella 488 personas, 161 de los cuales son infantes de marina (mapa $1 \mathrm{y}$ tabla 1).

Desde julio de 1991, se remiten esporádicamente al departamento de nefrología del Hospital Naval de Cartagena, infantes de marina procedentes de la isla de Manzanillo con un cuadro clínico de edema en piernas, disestesias y déficit motor en miembros inferiores, que no se relacionan con otros procesos clinicopatológicos; el 31 de diciembre de 1991 falleció un paciente que inició su cuadro clínico con la sintomatología descrita y cuya causa de muerte fue establecida como carditis.

Durante 1992 no se presentaron casos hasta julio, fecha a partir de la cual se comenzó a presentar una serie de 52 casos sospechosos de beriberi, 38 de ellos clasificados como probable y 20 que el grupo médico local consideró clásicos; el 9 de noviembre de 1992 falleció otro paciente que había iniciado, dos meses antes, una sintomatología similar a la de los otros pacientes, agravado con una insuficiencia cardíaca severa; el diagnóstico realizado fue de carditis.

En octubre de 1992 se remitió al Hospital Militar Central de Bogotá un paciente con impresión clínica de pericarditis, que respondió al tratamiento instaurado que incluyó antibióticos, tonicardiacos y una dieta bien balanceada. Dos meses antes había consultado por edema, disestesias y dificultades motoras. En este mes se solicitó ayuda para esclarecer el problema y se desplazó un equipo del INS que inició el estudio buscando establecer una posible etiología tóxica.

Entre enero y junio de 1993, consultaron 18 infantes de marina, ante lo cual se solicitó de nuevo ayuda y se organizó un equipo con investigadores del INS y OPS con la asesoría del $\mathrm{NIH}$ de Bethesda, EUA.

Los objetivos de la comisión fueron: 1) caracterizar la distribución de los casos, espacial y temporalmente; 2) determinar posibles causas y factores de riesgo; 3 ) definir si este problema corresponde a la llamada neuropatía epidémica estudiada por la misma época en Cuba; y 4) establecer medidas y las recomendaciones pertinentes para el control del brote.

Con base en la información revisada, se planteó como hipótesis que este cuadro clínico podría ser compatible con beriberi húmedo por deficiencia nutricional precipitada por ejercicio físico intenso y una dieta mal balanceada y rica en carbohidratos. 
Mapa 1. Isla de Manzanillo

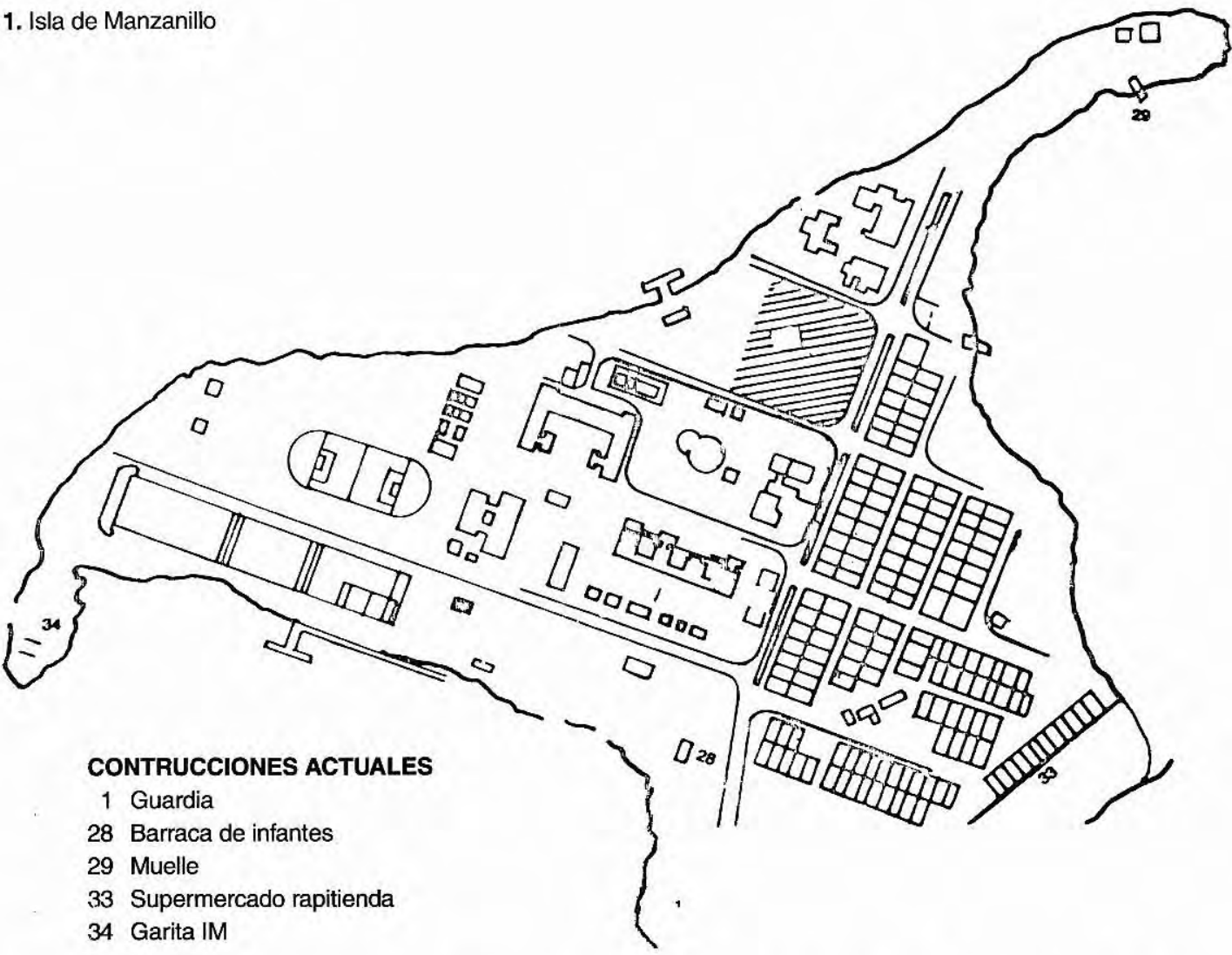

Tabla 1. Tasas de ataque y de letalidad en el brote de beriberi en Cartagena de Indias, Colombia, 1992-1993

\begin{tabular}{lrrccc}
\hline Población & $\mathbf{n}$ & Casos & TA\% & TM\% & TL\% \\
\hline Infantes & 161 & 22 & 13,6 & 1,2 & 9 \\
Cadetes & 141 & 0 & - & - & - \\
Oficiales & 20 & 0 & - & - & - \\
Familiares & 133 & 0 & - & - & - \\
Otros & 54 & 0 & - & - & - \\
Total & $\mathbf{4 8 8}$ & $\mathbf{2 2}$ & $\mathbf{4 , 5}$ & - & - \\
\hline
\end{tabular}

TA: tasa de ataque

TM. tasa de mortalidad

TL: tasa de letalidad

\section{Metodología}

Para explorar la hipótesis arriba mencionada, se procedió a realizar un estudio de casos y controles, para lo cual se elaboró un cuestionario que incluyó las siguientes variables: edad, sexo, tiempo de permanencia en la isla, lugar de procedencia, sitio donde prestaba guardia más frecuentemente, aplicación de medicamentos y vacunas, frecuencia de compañeros sexuales durante el último año, presencia de síntomas neurológicos durante el último año, antecedentes personales de atopias, otros sitios de la guarnición donde hubiera prestado servicio y frecuencia de consumo de pescado extraido de la bahía.

Se revisó la dieta en la escuela y se descartó la posibilidad de contaminación de los alimentos con aceites, solventes, rodenticidas y otros químicos. La dieta diaria del grupo afectado consistía básicamente de carbohidratos (yuca, arroz, pan, espagueti, papa y plátano); una a dos veces por semana se les servía huevo, carne o pescado.

Se realizaron mediciones de metales pesados en sangre, orina de 24 horas y en agua, de vitamina B1 en suero, para lo cual las muestras se procesaron y empacaron para ser transportadas refrigeradas hasta el laboratorio en el INS, de donde se enviaron alícuotas para estudio de 
niveles de tiamina en suero en la Universidad de Johns Hopkins (Baltimore, EUA).

Se realizó estudio de potenciales evocados; se practicó biopsia de nervio sural a cuatro infantes con diagnóstico confirmado, bajo aumento de 2,5 $\mathrm{X}$, tomando un fragmento de aproximadamente 5 $\mathrm{cm}$, que se dividió en dos partes iguales que se fijaron en formol al 4\% para estudio histopatológico; a los mismos infantes se les tomó muestras de LCR mediante punción lumbar atraumática.

Así mismo, se revisaron los registros de consulta externa con el fin de buscar casos sospechosos de beriberi no diagnosticados y se revisaron los estudios histopatológicos en material de autopsia de los pacientes que fallecieron.

Se procedió a interrogar y a realizar examen neurológico de 10 infantes incapacitados.

Infortunadamente omitimos investigar transcetolasa eritrocitaria y la cuantificación del piruvato en sangre, cuyo valor es, como se sabe, hasta $1,3 \mathrm{mg} / \mathrm{dL}$, cifra que usualmente está elevada en el beriberi húmedo.

Las definiciones de caso utilizadas fueron:

Caso sospechoso: todo residente de la isla que hubiese consultado por edema y parestesias en miembros inferiores a partir de enero de 1993.

Caso probable: caso sospechoso con confirmación clínica de compromiso sensitivo y motor, manifestado por hiporreflexia, hipoestesia y debilidad muscular en miembros inferiores.

Caso confirmado: caso probable que presentaba, además, disminución de amplitud de los potenciales evocados del nervio sural, con o sin evidencia de denervación en músculos tibial anterior o gemelos o con confirmación de neuropatía periférica en biopsia de nervio sural.

Se consideraron controles asintomáticos a infantes que no habían presentado edema o sólo habían presentado edema por debajo de la liga a partir de enero de 1993, con ausencia de síntomas de neuropatía periférica y reflejo aquiliano normal.

Se eligieron 22 casos y 22 controles para compararlos con respecto a la frecuencia de los factores de riesgo mencionados arriba. Las asociaciones se midieron usando las razones de disparidad (OR) y sus intervalos de confianza del 95\% (IC 95\%). Los datos se procesaron usando el programa Epi-Info 6.01, producido y distribuido por el CDC.

\section{Resultados}

Los estudios de muestras ambientales y biológicas procesadas durante 1992, fueron reportados así: los 35 sujetos a quienes se les estudió mercurio en sangre, presentaron niveles por debajo de 20 microgramos por litro (ppb); a los mismos se les estudió plomo en sangre, siendo reportados niveles por debajo de 0,038 $\mathrm{mg} / \mathrm{dL}(\mathrm{mg} / 100 \mathrm{~mL}$ ); se analizaron 23 muestras de orina de 24 horas para arsénico y talio, las cuales presentaron niveles máximos de $67 \mathrm{ppb}$ para arsénico y niveles por debajo de 0,2 miligramos por litro $(\mathrm{ppm})$ para talio; las tres muestras analizadas para mercurio, plomo, arsénico y talio en agua estuvieron por debajo de 0,5 microgramos por litro $(\mathrm{ppb})$ para mercurio, por debajo de 0,05 miligramos por litro (ppm) para plomo, por debajo de 20 microgramos por litro (ppb) para arsénico y menor de 0,2 ppm para talio, las dos muestras de agua de mar presentaron concentraciones de 40,32 y 0,36 ppm.

Los diez infantes interrogados y examinados neurológicamente por el grupo investigador tenían una edad entre 20 y 23 años (promedio 21), nueve procedentes de Barranquilla y uno de Medellín; tres de ellos comenzaron su enfermedad en noviembre de 1992 y siete entre abril y mayo de 1993; uno de los casos tratados durante 1992, presentó recaída en abril de 1993.

El cuadro clínico es relativamente estereotipado y se caracterizaba por la lenta aparición (más de una semana) de edema blando de pies que, en ocasiones, se extendía hasta la rodilla y que persistía entre 15 a 20 días, pérdida de peso, anorexia, adinamia, debilidad en las piernas, con disestesias (dolor en masa gemelar), parestesias en pies y manos (hormigueos y sensación de adormecimiento), dolor de los pies, especialmente después de la marcha y la permanencia de pie durante varias horas (el plantón). 
Síntomas menos frecuentes incluían taquicardia con el ejercicio, disnea con el trote, nicturia, sudoración excesiva de palmas y plantas, diarrea, constipación y trastornos del sueño. En ningún caso se informó fiebre o signos de infección.

En el examen neurológico, como hallazgos positivos se encontró disminución de sensibilidad en los pies con pérdida o disminución de la percepción del algodón, del pinchazo de la aguja o de la vibración del diapasón. Pocos casos presentaron ligera disminución de la fuerza muscular en el grupo tibial anterior, con dificultad para la marcha en talones. El examen de funciones nerviosas superiores, pares craneanos (incluyendo visión y audición) así como la coordinación y la marcha, fue normal en todos los casos.

El compromiso motor se iniciaba generalmente 15 días después y se manifestaba con debilidad muscular, reflejos disminuidos o abolidos y, ocasionalmente, pie caído. La recuperación ocurría con tratamiento vitamínico por vía oral, en forma gradual a partir de la octava semana; se recuperaban primero del compromiso motor y persistía más tiempo el compromiso sensitivo. Dos casos presentaron compromiso sensitivo distal de los miembros superiores.

Los exámenes de sangre y LCR fueron normales y las pruebas de neurofisiología clínica mostraron velocidades normales de conducción nerviosa motora y sensitiva, disminución de la amplitud de los potenciales sensitivos, especialmente del nervio sural y latencias distales normales. La electromiografía con aguja mostró, en algunos casos, evidencia de denervación con presencia de potenciales positivos.

Los niveles de tiamina en plasma se informaron como levemente bajos tanto en casos como en controles y oscilaron entre 3 y $12 \mathrm{ng} / \mathrm{mL}$ (normal entre 5 y $10 \mathrm{ng} / \mathrm{mL}$ ).

En total, se confirmaron 22 casos de beriberi húmedo de acuerdo con la definicion de caso confirmado, la mayoría de los cuales sólo tenían manifestaciones leves de neuropatía periférica nutricional; uno que inició su sintomatología durante 1991 falleció, 19 que iniciaron su sintomatología durante 1992 (uno falleció) y 2 que la iniciaron durante 1993. Dos personas que enfermaron el año anterior aún padecen secuelas de tipo sensitivo, consistentes en disestesias para ambos y limitación de la marcha en uno.

La tasa de ataque fue del $4,5 \%(22 / 488)$ y entre los infantes de marina del $13,6 \%(22 / 161)$; la tasa de mortalidad fue del 0,4\% (2/488) para los residentes de la isla y de $1,2 \%(2 / 161)$ entre los infantes de marina; la tasa de letalidad fue de $9 \%$ (2/22) (tabla 1).

En el estudio de casos y controles, se estableció que presentaban antecedentes de atopias respiratorias o dermatológicas el $24 \%$ de los participantes; esta característica estuvo presente en el $40 \%$ de los casos y en el $13 \%$ de los controles, con un OR de 4,44 y un valor de $p=0,06$; se evidencia una fuerte asociación entre tener 6 o más meses de estar en la isla de Manzanillo y padecer la enfermedad (tabla 2); también aparece como factor protector la ingestión de alimentos adicionales a la ración usual (tabla 3).

Los estudios histopatológicos en el corazón de los fallecidos mostraron edema, presencia de vacuolización en las fibras musculares, ocasional

Tabla 2. Estudios de casos y controles, brote de beriberi en Cartagena de Indias, Colombia, 1993

\begin{tabular}{lrrrrc}
\hline $\begin{array}{l}\text { Tiempo de } \\
\text { permanencia }\end{array}$ & Casos & Controles & Total & OR & IC 95\% \\
\hline$>6$ meses & 20 & 3 & 23 & 63,3 & $7,6-766,7$ \\
$<6$ meses & 2 & 19 & 21 & Referente \\
Total & 22 & 22 & $\mathbf{4 4}$ & & \\
\hline
\end{tabular}

Tabla 3. Estudio de casos y controles, brote de beriberi en Cartagena de Indias, Colombia, 1993

\begin{tabular}{lrrrrc}
\hline $\begin{array}{l}\text { Ingestión de Casos } \\
\text { otros }\end{array}$ \\
alimentos
\end{tabular}

OR=razón de disparidad 
necrosis de fibras aisladas, discreta fibrosis intersticial y perivascular con muy escasos polimorfonucleares, células redondas y cambios indicativos de hipertrofia (figuras 1 y 2); el estudio con microscopio electrónico a partir de material fijado en formol, comprueba y ubica el daño vacuolar y permiten, además, reconocer otras alteraciones organelares de la célula miocárdica (figuras 3 a 5). El proceso es compatible con beriberi húmedo.

Dos de las cuatro biopsias de nervio sural mostraron pérdida muy discreta de fibras mielinizadas gruesas y edema intersticial leve sin componente inflamatorio alguno (figura 6).

Es notoria la ausencia de contactos familiares con sintomatología parecida a la de los infantes; tampoco hay evidencia de un aumento en las consultas por un cuadro similar en la población general.

\section{Discusión}

El marco de referencia para considerar este brote como una forma de beriberi ha sido la clásica definición dada por varios autores ingleses: beriberi es una forma de neuritis periférica múltiple que se observa endémicamente o en forma epidémica en la mayoría de los climas tropicales o subtropicales y también bajo ciertas circuns-

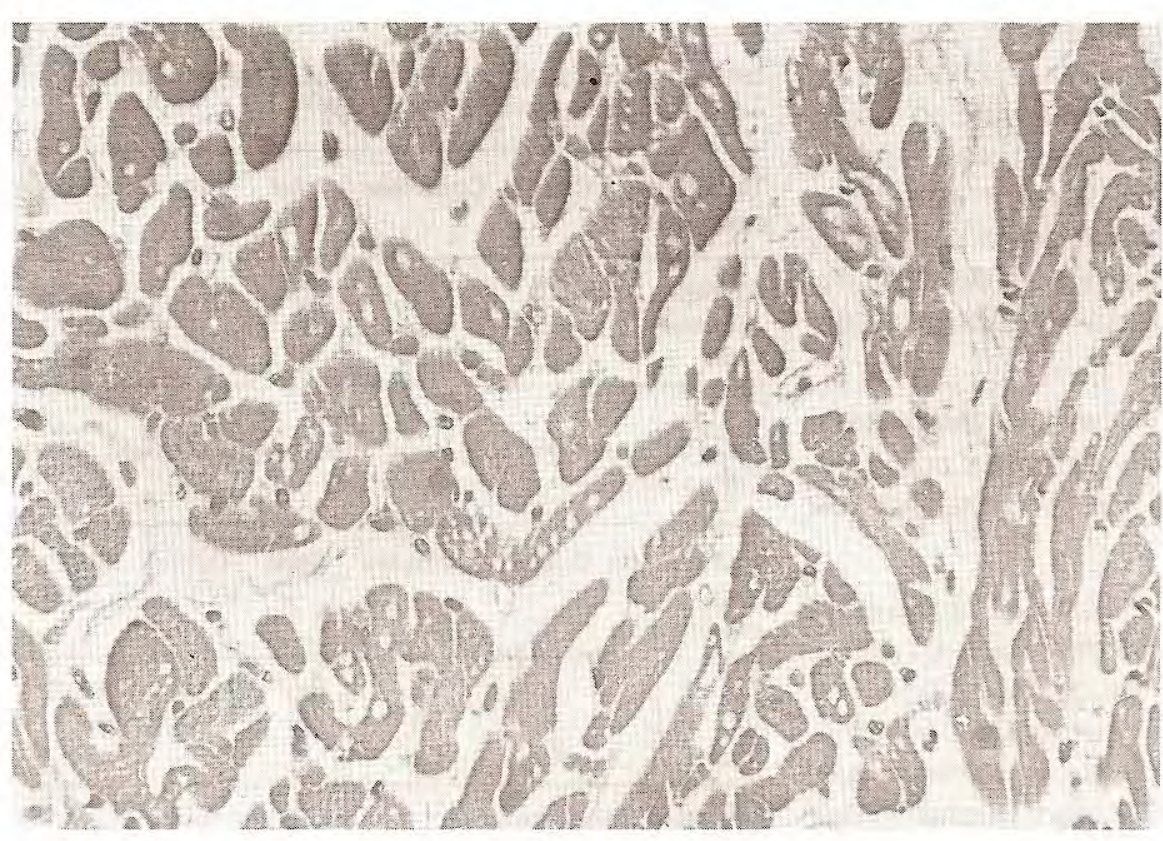

tancias en zonas más templadas. La mortalidad elevada suele ocurrir por insuficiencia cardiaca cuando predominan las manifestaciones cardiacas, observándose notoria dilatación del corazón relacionada con la elevación del piruvato y acompañando a la neuropatía periférica, usualmente de grado leve. Este cuadro severo es denominado beriberi húmedo o cardíaco. La falla cardiaca antes era denominada hipercinética y, en la actualidad, se llama de alto gasto.

Con base en los resultados de los exámenes de laboratorio, del examen neurológico de los diez infantes, así como de los resultados de los exámenes de neurofisiología clínica, se definió el caso epidemiológico como beriberi húmedo con leve componente de neuropatía periférica nutricional predominantemente sensitiva, con menor compromiso motor. Probablemente, la lesión corresponde a una neuropatía de tipo axonal, llamada tambien muerte axonal retrógrada (dying back neuropathy). Estas lesiones generalmente se deben a procesos de naturaleza nutricional, tóxica o metabólica o a una combinación de estos factores.

Entre las causas tóxicas se descartan insecticidas organofosforados, hexacarbonos aromáticos, nitrofuran y la intoxicación crónica por arsénico y talio.

Figura 1. Cambio vacuolar de las fibras miocárdicas con severo edema intersticial. Hipertrofia en algunas fibras. HE 250X. 


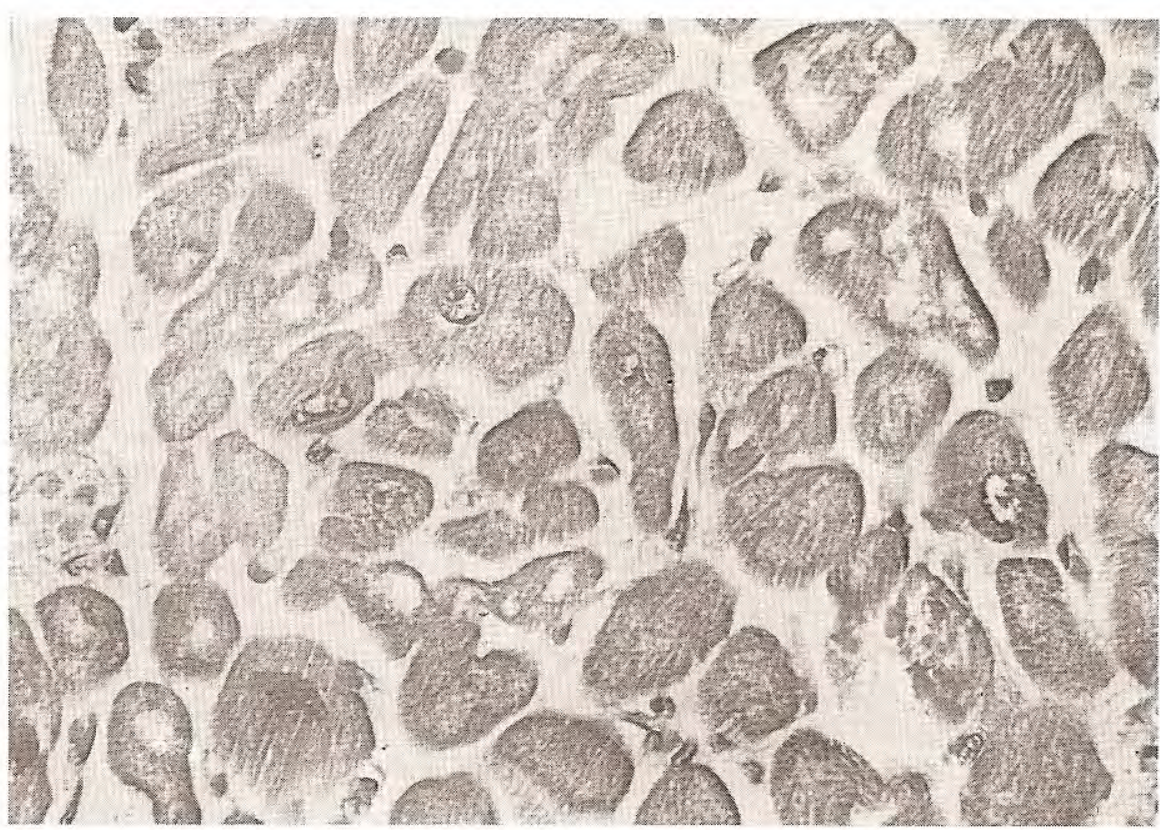

Figura 2. Los cambios descritos en la figura 1 son más evidentes a mayor aumento. HE 450X.

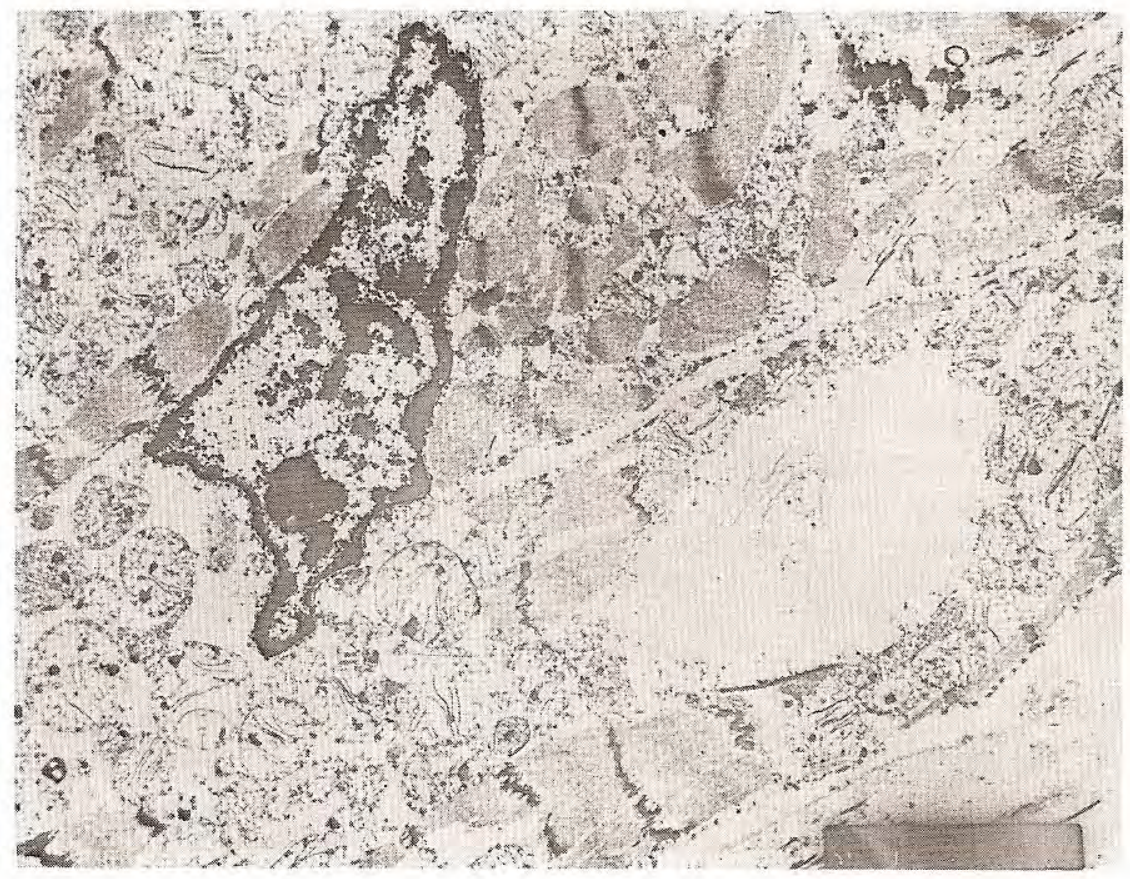

Figura 3. Fibra cardiaca con amplia zona clara, vacía del citoplasma en la cual han desaparecido las miofibrillas. El núcleo es picnótico y las mitocondrias son edematosas. 


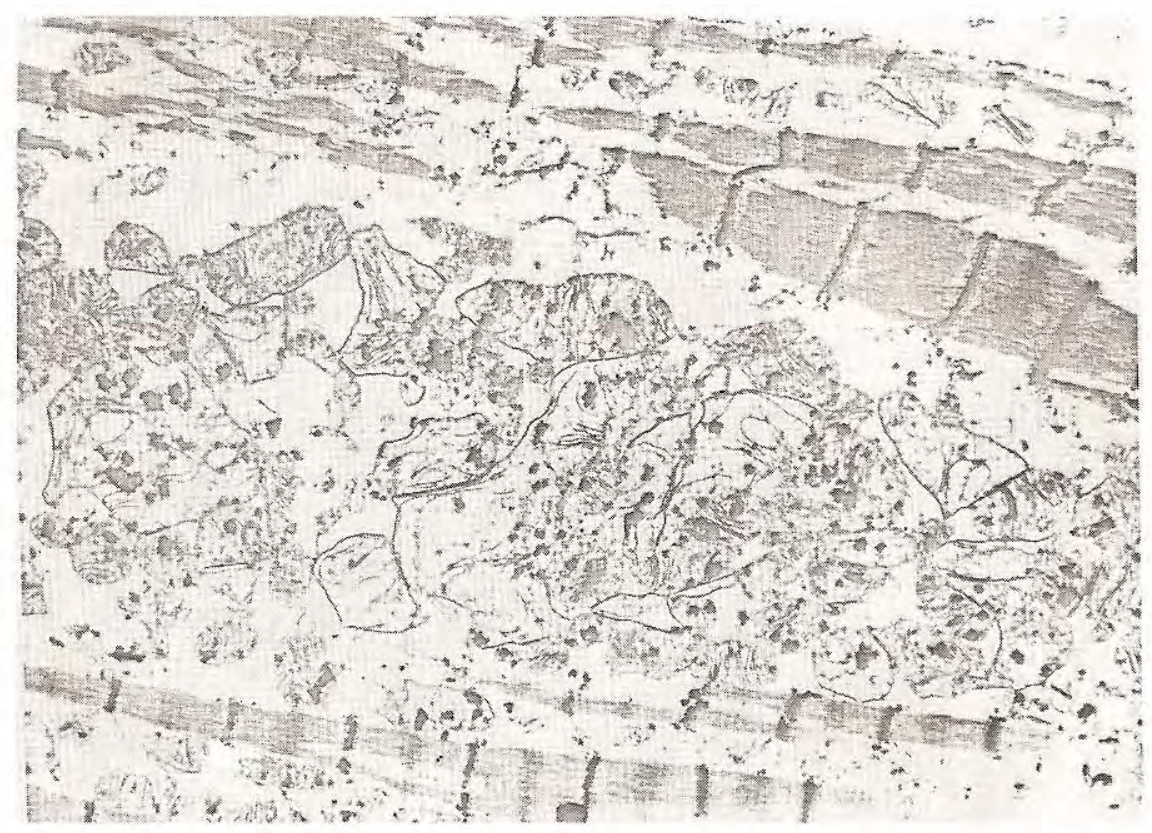

Figura 4. Acúmulo intermiofibrilar de mitocondrias edematosas con cuerpos densos, indicadores de muerte celular. Se aprecia, también, edema del citoplasma, evidenciado por las áreas claras y ruptura de miofibrillas, arriba, en el centro. La validez de estos hallazgos, dada las condiciones de preservación del tejido, está sujeta a discusión.

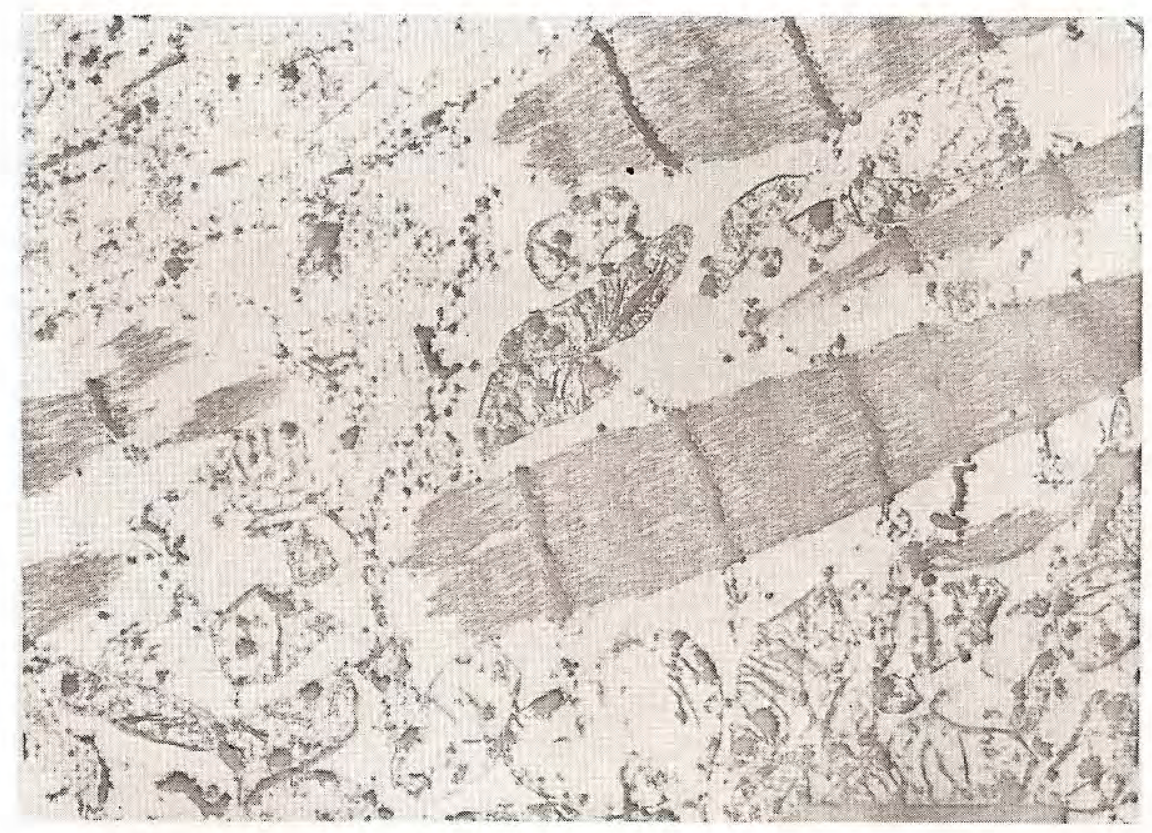

Figura 5. Parte de una fibra cardiaca con citoplasma edematoso y fragmentación de las miofibrillas. El edema y los cuerpos densos mitocondriales son indicadores de muerte celular. 


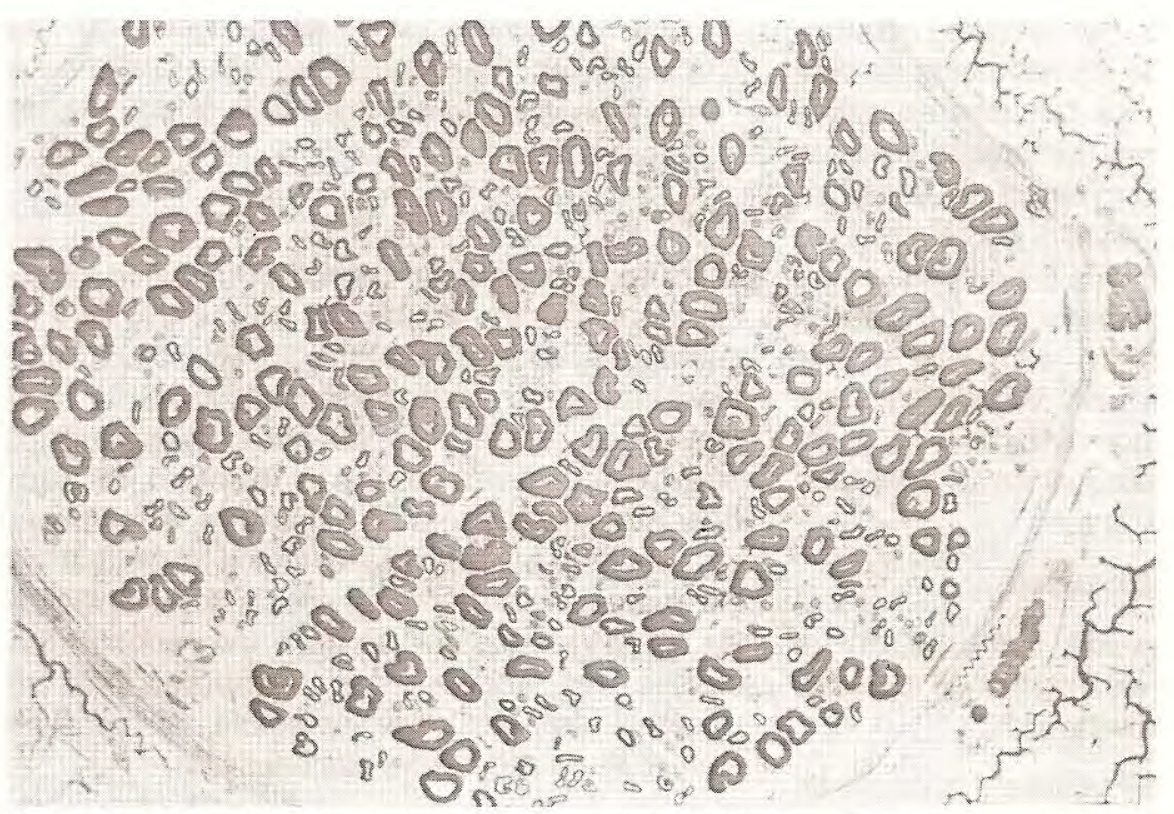

Figura 6. Fascículo del nervio sural: edema intersticial leve sin componente celular inflamatorio; hay discreta disminución de fibras mielínicas gruesas. Fijación en glutaraldehídoácido ósmico. Azul de toluidina 160X.

Los hallazgos clínicos y de laboratorio sugestivos, los de histopatología muy evidentes y enteramente compatibles, el desbalance dietético y los estudios epidemiológicos establecen el déficit de tiamina como la causa de este grave problema ocurrido en la isla de Manzanillo, con la ocurrencia de dos casos fatales de beriberi húmedo en dos jóvenes de 21 años de edad, fallecidos, uno el 31 de diciembre de 1991 (A-192) y el otro el 9 de noviembre de 1992 (A-10-92) con cuadros de insuficiencia cardiaca congestiva rápidamente fatal, sin respuesta al tratamiento convencional con furosemida, lanicor y dopamina.

\section{Conclusiones}

Se trata de una enfermedad potencialmente letal que se presenta como un cuadro mixto de falla cardiaca y neuropatía leve periférica frecuentemente, con gran capacidad para causar brotes, observándose susceptibilidad en personas institucionalizadas que practican ejercicios fuertes, luego de seis meses de permanencia.

Hasta donde se ha podido establecer es el primer brote de beriberi húmedo estudiado desde
1933, en Colombia (1). Es probable que algunos casos hayan pasado desapercibidos.

Es claro que, en estos momentos, el brote de la enfermedad está básicamente confinado a la isla de Manzanillo y dentro de ésta sólo a los infantes de marina; el examen clínico y epidemiológico realizado plantean, en primera instancia, a la interacción de factores nutricionales y ambientales como etiología del cuadro. Desde el punto de vista nutricional, el déficit de tiamina es la explicación más plausible, apoyado por el hallazgo de una dieta demasiado rica en carbohidratos hasta hace muy poco; además, la probable presencia de tiaminasas en el pescado que forma parte de la dieta cotidiana de algunos enfermos y los niveles de talio hallados en algunos de ellos pueden haber precipitado tal déficit.

Es necesario resaltar la efectividad del método epidemiológico, que en este caso aportó los elementos faltantes y orientó el estudio de los casos y del brote, ofreciendo oportunas recomendaciones que sirvieron para controlar el problema. Estas recomendaciones consistieron fundamentalmente en un cambio dietético, en la administración de complejo $\mathrm{B}$ y en la disminución 
drástica del consumo de alcohol, hábito detectado en la mayoría de los miembros de este grupo. Estas decisiones se basaron en experiencias previas que ya son numerosas y en los resultados del estudio monumental de Takaki en la armada japonesa de la cual subrayamos lo siguiente: en 1878 entre 4.528 soldados hubo 4.485 enfermos de beriberi, de los cuales murieron 32. Nueve años después y tras instaurar una dieta rica en proteínas que, además, cambió el arroz por verduras y leche, se logró que en 1887 no se presentara un solo caso de beriberi entre 9.106 soldados.

\section{Recomendaciones a corto plazo}

- Incluir suplementos de tiamina en la alimentación diaria de los infantes de marina y cadetes de la isla de Manzanillo.

- Prohibición absoluta de pescar y bañarse en las aguas que rodean la isla de Manzanillo, dado los altos niveles de talio encontrados en estas aguas.

- Exámenes oftalmológicos, auditivos, valoración de sensibilidad, motricidad y pruebas de función cardiaca a todos los casos sospechosos de esta patología.

\section{Recomendaciones a mediano y largo plazo}

- Recanalización del caño Zapatero y vigilancia ambiental de los agentes químicos y orgánicos vertidos a la bahía.

- Capacitación en epidemiología y vigilancia en salud pública a los médicos de la fuerza naval, dada la debilidad de este componente en la formación de los médicos generales.

- Control nutricional sobre los alimentos que provienen de la calle y van a surtir los casinos de infantes y cadetes.

- Mejoría de los protocolos de autopsia e institucionalizar las discusiones clinicopatológicas alrededor de los casos fallecidos dentro del Hospital Naval.

- Organizar la vigilancia en salud pública para el subsistema de salud de las fuerzas armadas para detectar e intervenir rápida y eficazmente los diferentes daños a la salud que se presenten.
- Añadir tiamina IV al tratamiento de los pacientes con falla cardiaca que no respondan en forma adecuada al tratamiento convencional.

\section{Adendum}

Mientras este artículo estaba en revisión editorial, ocurrió un brote de una enfermedad semejante en el departamento de Bolívar, que afectó un grupo de 30 personas jóvenes de sexo masculino, entre 18 y 28 años, cuyo cuadro clínico fue sensiblemente idéntico al aquí descrito; dos de estos pacientes fallecieron, aparentemente, con falla cardiaca y, en una de las autopsias, el cuadro de miocardiopatía vacuolar es evidente. Se aplicaron las recomendaciones que aquí se han formulado con un resultado satisfactorio e inmediato, tal como se describirá en un informe posterior (31).

\section{Agradecimientos}

Al capitán Víctor Manuel Villalba, director del Hospital Naval, Cartagena; al capitán Mario Rubiano Groot, Escuela de Suboficiales, Barranquilla; al teniente Rogelio Botero, jeie de la COAL, ENAP, Organización Panamericana de la Salud. Al profesor Alfonso Villamil Bernal por su revisión y corrección, y por sus muy autorizadas y enriquecedoras sugerencias.

\section{Bibliografía}

1. Sotomayor H. Historia sanitaria del conflicto amazónico colombo-peruano 1932-1934. Santa Fé de Bogotá: Fondo Rotatorio de la Policía Nacional,1994:49.

2. Victor M, Adams RD, Collins GH. The Wernicke Korsakoff syndrome and related neurologic disorders due to alcoholism and malnutrition. Second edition, Philadelphia: F. A. Davis Company, 1995.

3. OPS. Neuropatía epidémica en Cuba. Boletín epidemiológico 1993:14(2).

4. Gay J, Porrata C, Hernández M y col. Factores dietéticos de la neuropatía epidémica en la isla de la Juventud, Cuba. Bol Oficina Sanit Panam 1994: 117(5):389-9.

5. Toro G. Hambre, hombre y contaminación del medio ambiente. Rev Fac Med U Nal de Colombia 1993:41:28 -45 .

6. Harper C. The incidence of Wernicke's encephalopaty in Australian neuropathological study of 131 cases. $J$ Neurol Neurosurg Psychiat 1983;46:593-8. 
7. Leigh D. Subacute necrotizing encephalomyelopaty in an infant. Neurol Neurosurg Psychiat 1951;14:216-21.

8. Victor M, Yakovlev PI. SS Korsakoff's psychic disorder in conjunction with peripheral neuritis. A translation of Korsakoff's original article with brief comments on the author and his contributions to clinical medicine. Neurology 1955;5:394-406.

9. Dreyfus PM. Ambliopia and other neurological disorder associated with chronic alcoholism. In: Vinken PJ, Bruyn GW, editors: Handbook of clinical neurology, vol. 28, Metabolic and deficiency diseases on the nervous system, Part II. Amsterdam: North Holland Publishing Co., 1976:331-47.

10. Fisher $\mathbf{C H}$. Residual neuropathological changes in $\mathrm{Ca}$ nadians held prisoners of war by the Japanese. Can Ser Med J 1955;11:157-99.

11. Takahashi J, Nakamura H. Axonal degeneration in beriberi neuropathy. Arch Neurol 1976;33: 836-41.

12. Victor M. Polyneuropathy due to nutritional deficiency and alcoholism. In: Dyck PJ, Thomas PK, Lambert EH, Bunge R, editors: Peripheral neuropathy, vol 2, 2nd. ed. Philadelphia: W. B. Saunders Co., 1984:1899-940.

13. Cruickshank EK. Painful feet in prisoners of war in the Far West: review of 500 cases. Lancet 1946;2:369-72.

14. Román G, Spencer PS, Schoemberg BS. Tropical myeloneuropathies: the hidden endemias. Neurology 1985;35:1158-70.

15. Takahashi K. Thiamine deficiency neuropathy: a reappraisal. Int J Neurol 1981;15:245-53.

16. Djoenaidi W, Notermans SL, Dunda G. Beriberi cardiomyopathy. Eur J Clin Nutr 1992;46(3):227-34.

17. Velloso LG, Barreto AC. Beriberi heart disease. Arg Bras Cardiol 1991;56(3):235-7.

18. Danielson C, Joyce S. Beriberi heart disease in a schizophrenic with an unusual diet. $J$ Emerg $M e d$ 1990;8(6):705-7.

19. Phua K, Goh LG, Koh K, Ong CN, Wong ML, Lee HP. Thiamine deficiency and sudden deaths: lessons from the past. Lancet 1990;335 (8703):1471-2.
20. Lonsdale $\mathbf{D}$. Thiamine deficiency and sudden deaths. Lancet 1990;336(8711):376.

21. Lamont NM. Cardiomyopathy or cardiac beriberi in sugarcane field workers. S Afr Med J 1990;77(10):542.

22. Naidoo DP, Gathiram V, Sadhabiriss A, Hanssen F. Clinical diagnosis of cardiac beriberi. $S$ Afr Med $J 1990$; 77(3):125-7.

23. Cappelli V, Bottinelli R, Polla B, Reginiani C. Altered contractile properties of rat cardiac muscle during experimental thiamine deficiency and food deprivation. $J$ Mol Cell Cardiol 1990;22(10):1095-106.

24. Barrett TG, Forsyth JM, Nathavitharana KA, Booth IW. Pothentially lethal thiamine deficiency complicating parenteral nutrition in children. Lancet 1993;341 (8849):901.

25. Lange R, Erhard J, Eigler FW, Roll C. Lactic acidosis from thiamine deficiency during parenteral nutrition in a two year old boy. Eur J Ped Surg 1992;2(4):241-4.

26. Zak J, Burns D, Lingenfelser T, Steyn E, Marks IN. Unusual complication of prolongued parenteral nutrition. JPEN. Parenter Enteral Nutr 1991;15(2):200-1.

27. Van-Zaanen HC, Van-der-Lelie J. Thiamine deficiency in hematologic malignant tumors. Cancer 1992;69(7): 1710-3.

28. Fujita Y, Sata T, Gondo K, Fukazawa M, Kukita J, Zaitsu A, Yoshitake J, Ueda K. Cardiac beriberi (shoshin beriberi) caused by excessive intake of isotonic drink. Acta Paediatr Jpn 1992;34(4):466-8.

29. Martin PR, McCool BA, Singleton CK. Genetic sensitivity to thiamine deficiency and development of alcoholic brain disease. Alcohol Clin Exp Res 1993;17(1): 31-7.

30. Butterworth RF, Gaudreau V, Vincelette J, Bourgault AM, Lamthe F, Nutini AM. Thiamine deficiency in AIDS (Letter, comment), Lancet 1991;338(8774):1086.

31. Merchán PO. Comunicación personal. 